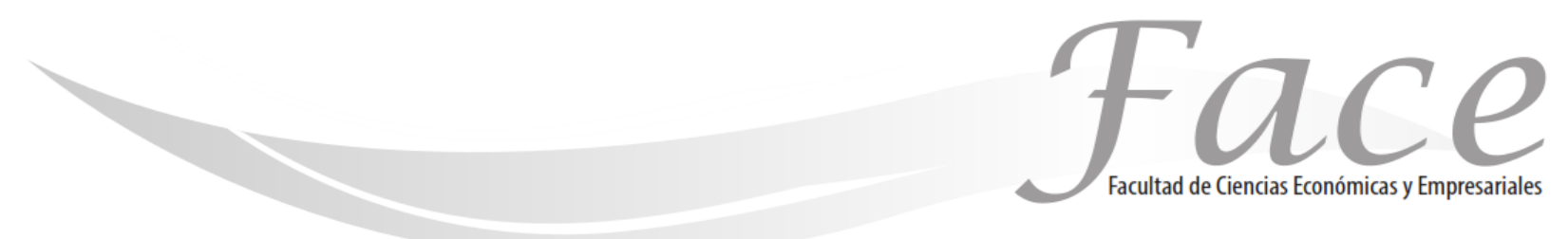

ISSN Impreso: 1794-9920

ISSN Electrónico: 2500-9338

Volumen $20-\mathrm{N}^{\circ} 1$

Año 2020

Págs. $110-124$

(c) (†)

\title{
EVALUACION DEL IMPACTO DE LOS FACTORES DE RIESGO QUE GENERAN ENFERMEDADES PROFESIONALES Y ACCIDENTES LABORALES EN LAS PYMES DEL SECTOR ELÉCTRICO DE LA CIUDAD DE CÚCUTA-COLOMBIA
}

Fecha de Recepción: Febrero 17 de 2020

Fecha de Aprobación: Junio 1 de 2020

\author{
Gustavo Enrique Bohórquez Mantilla * \\ ORCID: https://orcid.org/0000-0003-1727-7770. \\ Javier Mauricio García Mogollón ** \\ ORCID: https://orcid.org/0000-0001-7423-8909
}

Resumen:

La presente investigación tiene como punto de partida la identificación de las tasas de accidentalidad y enfermedades profesionales presentes a nivel internacional, nacional y regional, que se presentan en los lugares de trabajo, por medio de publicaciones significativas como la organización internacional del trabajo, ministerio de trabajo de Colombia, las administradoras de riesgos laborales en Colombia y estudios realizados en sectores económicos de Colombia. Por consiguiente como solución a dicha problemática la investigación tuvo como objetivo proponer lineamientos estratégicos de mejora para minimizar el impacto de los factores de riesgo que generan enfermedades profesionales y accidentes en las PYMES del sector eléctrico de la ciudad de Cúcuta, mediante un estudio descriptivo por segmentos cuya población objetivo estuvo conformada por 36 supervisores y 84 trabajadores vinculados respectivamente a 36 pequeñas y medianas del empresa del sector eléctrico, en la cual se aplicaron dos cuestionarios de percepción y encuesta al marco poblacional. En la identificación de los factores de riesgos, el riesgo físico, químico, biológico, eléctrico, mecánico, disergonómico y psicosocial tiene poca presencia o inexistencia en el lugar de trabajo, debido a que actualmente las PYMES del sector eléctrico cuentan con sistemas de gestión de seguridad y salud ocupacional que permiten condiciones saludables a los trabajadores. Sin embargo, es importante proponer como acción de mejora continua los lineamientos estratégicos de mejora para las PYMES así: Estrategia No 1. Jornadas de concientización, sensibilización y motivación, Estrategia No 2. Talleres de capacitación y adiestramiento, Estrategia No 3. Implementación del sistema de seguridad y salud ocupacional, y Estrategia No 4. Sistematización del proceso de evaluación. Puede aducirse, que las PYMES del sector eléctrico, aunque a la fecha no presentan casos de accidentalidad ni enfermedades profesionales deben implementar acciones para mantener en los sistemas de gestión de seguridad y salud ocupacional.

Palabras clave: Pequeñas y medianas empresas (PYMES), Factor de riesgo, accidentes laborales, enfermedades profesionales.

\footnotetext{
* Magister Gestión Ingeniería Industrial, Especialista en Empresas, Ingeniero Industrial. Docente e investigador de la Universidad de Pamplona, Colombia. Contacto: gbohorquez@unipamplona.edu.co

* Doctor en Ciencias Gerenciales, Magister en Ciencias Gerenciales, Administrador de la Universidad de Pamplona. Docente tiempo Completo de la Universidad de Pamplona, investigador del Grupo GICEE de la Universidad de Pamplona. Contacto: jmgmogollon@unipamplona.edu.co
} 


\title{
ENVIRONMENTAL ACCOUNTING AND ITS SCIENTIFIC PRODUCTIVITY THROUGH BIBLIOMETRY
}

\begin{abstract}
:
In the identification of risk factors, physical, chemical, biological, electrical, mechanical, dysergonomic and psychosocial risk has little or no presence in the workplace, because currently SMEs in the electricity sector have management systems for occupational health and safety that allow healthy conditions for workers. However, it is important to propose as continuous improvement action the strategic improvement guidelines for SMEs as follows: Strategy No. 1. Awareness, awareness and motivation sessions, Strategy No. 2. Training and education workshops, Strategy No. 3. System implementation occupational health and safety, and Strategy No. 4. Systematization of the evaluation process. It can be argued that SMEs in the electricity sector, although to date there are no cases of accidents or occupational diseases, must implement actions to maintain occupational health and safety management systems.
\end{abstract}

Keywords: Small and medium-sized enterprises (SMEs), Risk factor, occupational accidents, occupational diseases.

\section{CONTABILIDADE AMBIENTAL E SUA PRODUTIVIDADE CIENTÍFICA ATRAVÉS DA BIBLIOMETRIA}

\begin{abstract}
Resumo:
Esta pesquisa tem como ponto de partida a identificação das taxas de acidentes e doenças ocupacionais presentes a nível internacional, nacional e regional, as quais são apresentadas no ambiente de trabalho, através de publicações significativas como a organização internacional do trabalho, ministério da trabalho na Colômbia, gestores de riscos trabalhistas na Colômbia e estudos realizados em setores econômicos na Colômbia.

Portanto, como solução para este problema, o objetivo da pesquisa foi propor diretrizes estratégicas de melhoria para minimizar o impacto dos fatores de risco que geram doenças ocupacionais e acidentes em PMEs do setor elétrico da cidade de Cúcuta, por meio de um estudo descritivo por segmentos. A população-alvo foi composta por 36 supervisores e 84 trabalhadores vinculados, respectivamente, a 36 pequenas e médias empresas do setor elétrico, nos quais foram aplicados dois questionários de percepção e um inquérito ao quadro populacional. Na identificação dos fatores de risco, o risco físico, químico, biológico, elétrico, mecânico, disergonômico e psicossocial tem pouca ou nenhuma presença no ambiente de trabalho, pois atualmente as PMEs do setor elétrico possuem sistemas de gestão para segurança e saúde ocupacional que possibilitem condições de saúde aos trabalhadores. No entanto, é importante propor como ação de melhoria contínua as diretrizes de melhoria estratégica para as PMEs como segue: Estratégia $n^{0} 1$. Sessões de conscientização, conscientização e motivação, Estratégia $n^{0} 2$. Oficinas de treinamento e educação, Estratégia $n^{0} 3$. Implementação do sistema segurança e saúde ocupacional e Estratégia $n^{0} 4$. Sistematização do processo de avaliação. Pode-se argumentar que as PMEs do setor elétrico, embora até o momento não haja casos de acidentes ou doenças ocupacionais, devem implementar ações para manter os sistemas de gestão da segurança e saúde ocupacional.
\end{abstract}

Palavras-chave: As pequenas e médias empresas (PME), fatores de risco, acidentes de trabalho, doenças ocupacionais 


\section{INTRODUCCIÓN:}

Como necesidad prioritaria el hombre está impulsado a trabajar; sin embargo, cada una de las funciones laborales que realiza según su especialidad, está inmersa en un ambiente propio que genera ciertas condiciones, que hacen, que un trabajador se exponga en forma individual o colectiva a determinados factores de riesgo, los cuales propician la presentación de accidentes laborales o enfermedades profesionales.

Así pues, la Organización Internacional del Trabajo (OIT, 2000) refiere a que el lugar de trabajo, los peligros para la salud y la seguridad laboral de los individuos están relacionados con la naturaleza del trabajo que ejecuta.

Del mismo modo los trabajadores del sector eléctrico, que trabajan en instalaciones de redes de media y baja tensión, se ven expuestos a factores de riesgos tales como: físicos, bilógicos, químicos, eléctricos, disergonómicos, mecánicos y psicosociales, que pueden desencadenar trastornos de salud y a su vez repercusiones negativas tanto en la empresa como a nivel personal, tales como ausentismo laboral, déficit en el proceso del trabajo, indemnizaciones, entre otras.

Uno de los principales estudios de investigación en el sector eléctrico, publicados en el ámbito internacional, hace referencia a los factores de riesgo ocupacional y trastornos de salud en los linieros, Norka (2010). Así mismo en el ámbito nacional se hace referencia a un estudio sobre las causas mortales en trabajo en altura en Colombia, Tellez (2009). Este último indica la importancia de la prevención de eventos mortales en las maniobras de trabajos en altura en el sector eléctrico.

Por tal motivo, el propósito general de esta investigación fue proponer los lineamientos estratégicos de mejora para minimizar el impacto de los factores de riesgos que generan enfermedades profesionales y accidentes laborales en las pequeñas y medianas empresas (PYMES) del sector eléctrico de la ciudad de Cúcuta.
De acuerdo a lo anterior, los resultados obtenidos de esta investigación sirven para establecer acciones correctivas dirigidas a los factores de riesgos laborales a los que se encuentran expuestos los trabajadores de las PYMES del sector eléctrico de la ciudad de Cúcuta, ya que las enfermedades y accidentes laborales pueden ser prevenibles, contribuyendo de esta manera a mejorar las condiciones de trabajo, por lo tanto, se reflejará en su salud y seguridad laboral. El objetivo de este trabajo fue Proponer lineamientos estratégicos de mejora para minimizar el impacto de los factores de riesgo que generan enfermedades profesionales y accidentes en las pymes del sector eléctrico de la ciudad de Cúcuta.

Se tuvo presente para este proyecto una referencia planteada por García, Malagón-Sáenz, y GarcíaMogollón. que dan a conocer en su trabajo aspectos relevantes como: innovación tecnológica, la mejora continua, gerencia estratégica y gestión del recurso humano como parte de su competitividad, esta variables presentes para lograr llegar a generar parte de los resultados del artículo.

\section{METODOLOGÍA:}

En el caso que nos ocupa del trabajo de investigación y en función de los objetivos planteados, éste se enmarca dentro de los diseños de campo no experimentales que permitirán recolectar la información directamente de la realidad estudiada para proceder luego al análisis e interpretación, por lo cual se utilizó el método de la encuesta y como herramienta de recolección de información el cuestionario.

Según Danke, citado por Hernandez, Fernandez y Baptista (1998) la investigación descriptiva es "la investigación que busca especificar propiedades importantes de personas, grupos, comunidades 0 cualquier otro fenómeno que sea sometido a análisis" : Dentro de esta investigación se realizó una investigación descriptiva, se realizó una investigación de campo: esto se refiere a aquella investigación que se realiza en el lugar y tiempo en que ocurren los fenómenos objetos de estudio (Zorilla, 1990), para este 
EVALUACION DEL IMPACTO DE LOS FACTORES DE RIESGO QUE GENERAN ENFERMEDADES PROFESIONALES Y ACCIDENTES LABORALES EN LAS PYMES DEL SECTOR ELÉCTRICO DE LA CIUDAD DE CÚCUTA-COLOMBIA

Gustavo Enrique Bohórquez Mantilla - Javier Mauricio García Mogollón

caso las PYMES del sector eléctrico del Norte de Santander.

En el enfoque cuantitativo generalmente se elige una idea, la cual transforma en preguntas de investigación, que luego derivan en hipótesis. Desarrolla un plan para probar estas hipótesis, midiendo las variables en un determinado contexto. Con frecuencia hace uso de la estadística para el análisis de las mediciones obtenidas y a partir de ahí establece conclusiones respecto a las hipótesis formuladas. Según Pita Fernández (2000). De acuerdo a lo expuesto anteriormente y al diseño de la investigación y sus objetivos considero que el enfoque de la misma es cuantitativo.

La población es el conjunto de todos los casos que concuerdan con una serie de especificaciones (Selltiz et al., 1980). La población debe situarse claramente en torno a sus características de contenido, de lugar y en el tiempo.

Weiers (1986), señala que "La población es el total de elementos sobre la cual queremos hacer una inferencia basándonos en la información relativa a la muestra" (p.97). Y entendiéndose por inferencia de acuerdo al diccionario de la real academia española como la deducción de una cosa a partir de otra. Las poblaciones de estudio en la presente investigación se consideraron finitas y quedaron definidas como:

1. Población $N^{0} 1$ corresponde a los gerentes 0 supervisores HSEQ pertenecientes a las PYMES del sector eléctrico de la ciudad de Cúcuta Norte de Santander.

2. Población $\mathrm{N}^{0} 2$ se refiere a los trabajadores pertenecientes a las PYMES del sector eléctrico de la ciudad de Cúcuta Norte de Santander.

La muestra es un subgrupo de la población. Se puede decir que es un subconjunto de elementos que pertenecen a ese conjunto definido en sus características al que se llama población, (Hernández Sampieri, 2006).

Para la realización del estudio se ha decidido calcular dos muestras mediante la formula propuesta por Weires (1996). La muestra 1 esta conformada solo por Supervisores del Sistema de Gestión de Seguridad y Salud Ocupacional que posee la empresa, y la muestra 2 conformada por los Supervisores encargados de
Gestionar el Sistema de Gestión de Seguridad y Salud Ocupacional y por los Trabajadores Electricidad. La muestra 1 se le aplicara al cuestionario II y a la muestra 2 se le aplicara el cuestionario I.

Según Hernández Sampieri, (2006). Una vez seleccionado el diseño de la investigación y determinada la muestra adecuada, la siguiente etapa consiste en la recolección de los datos pertinentes sobre los atributos, conceptos, cualidades o variables de los participantes, casos, sucesos, comunidades u objetos involucrados en la investigación.

En esta investigación se aplicará un cuestionario a los gerentes o supervisores HSEQ y a los empleados de las PYMES del sector eléctrico de la ciudad de Cúcuta Norte de Santander, además. Esto con el fin de desarrollar un plan de mejora organizacional para minimizar el impacto de los factores de riesgos que generan enfermedades profesionales y accidentes laborales.

El instrumento de medición empleado para esta investigación, es el cuestionario que está diseñado para ser aplicado a los responsables o supervisores encargados de las funciones de Seguridad y Salud Ocupacional y a los Trabajadores Electricistas de las organizaciones. Las empresas pertenecen al ramo industrial de servicio de mantenimiento eléctrico por contrato. En esta investigación se elaboraron 2 cuestionarios; uno dirigido a medir e identificar los factores de riesgo en la empresa y el otro buscar evaluar el Sistema de Gestión de Seguridad y Salud Ocupacional e Impacto Socioeconómico.

La información obtenida con estos cuestionarios, posteriormente será empleada para realizar un análisis que permita medir dichos aspectos. Las preguntas los cuestionarios son de tipo cerrada en escala Likert unipolar de cinco alternativas, en ellas se les pide a los encuestados expresaran su opinión sobre aspectos y elementos que especifican los riesgos presentes en los ambientes laborales y las condiciones de la organización de la Gestión del Sistema de Seguridad y Salud Ocupacional e Impacto Socioeconómico.

El cuestionario I está estructurado en preguntas que agrupan los riesgos específicos. Estos grupos son: el grupo I mide los riesgos Físicos, el grupo II se refiere 
al riesgo Químico, el grupo III mide los riesgos Biológicos, el grupo IV los riesgos Mecánicos. El Grupo $\mathrm{V}$, mide la presencia de los riesgos Disergonómicos el Grupo VI mide los riesgos Eléctricos y el Grupo VII los riesgos Psicosociales. Ver tabla 1.

\section{Tabla Nº 1 Relación grupos Vs. Ítems}

\begin{tabular}{|c|c|c|c|}
\hline OBJETIVO & GRUPOS DE VARIABLES & CUESTIONARIO & ÍTEMS CUESTIONARIO \\
\hline \multirow{7}{*}{$\begin{array}{ll}\text { IDENTIFICACIÓN DE } \\
\text { FACTORES } \\
\text { RIESGO }\end{array}$} & $\begin{array}{l}\text { Grupo I } \\
\text { RIESGO FísICO }\end{array}$ & \multirow[t]{7}{*}{ 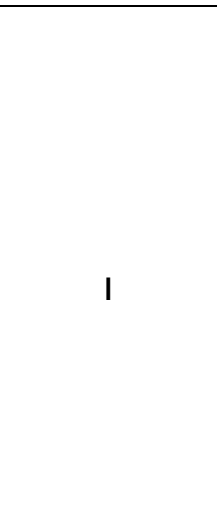 } & 1 a 14 \\
\hline & $\begin{array}{l}\text { Grupo II } \\
\text { RIESGO QUÍMICO }\end{array}$ & & 15 a 25 \\
\hline & $\begin{array}{l}\text { Grupo III } \\
\text { RIESGO BIOLÓGICO }\end{array}$ & & 26 a 32 \\
\hline & $\begin{array}{l}\text { Grupo IV } \\
\text { RIESGO MECÁNICO }\end{array}$ & & 33 a 47 \\
\hline & $\begin{array}{l}\text { Grupo V } \\
\text { RIESGO DISERGONOMICO }\end{array}$ & & 48 a 59 \\
\hline & $\begin{array}{l}\text { Grupo VI } \\
\text { RIESGO ELÉCTRICO }\end{array}$ & & 60 a 73 \\
\hline & $\begin{array}{l}\text { Grupo VII } \\
\text { RIESGO PSICOSOCIAL }\end{array}$ & & 74 a 84 \\
\hline \multirow{2}{*}{$\begin{array}{l}\text { SGSYSO / IMPACTO } \\
\text { SOCIAL } \\
\text { ECONÓMICO }\end{array}$} & $\begin{array}{l}\text { Grupo VIII } \\
\text { SGSYSO }\end{array}$ & \multirow{2}{*}{ II } & 1 a 19 \\
\hline & $\begin{array}{l}\text { Grupo IX } \\
\text { ACCIDENTABILIDAD Y CONDICIONES DE } \\
\text { SALUD DE TRABAJADORES E IMPACTO } \\
\text { SOCIAL }\end{array}$ & & 20 a 42 \\
\hline
\end{tabular}

Fuente: Elaboración propia.

\section{RESULTADOS Y DISCUCIÓN:}

En los anexos 8 y 9 se presentan el documento estadísticos descriptivos por segmento, en donde se refleja la importancia para cada segmento, de cada ítem, ordenados de forma decreciente, En este se puede apreciar los ítems más relevantes. También en los anexos 6 y 7 , contienen la información Resumen de Casos por Segmento, ordenados según los distintos ítems considerados en nuestro cuestionario.

\subsubsection{ANÁLISIS ESTADÍSTICO SEGMENTO TRABAJADOR ELECTRICISTA}

En el Tabla 2, se presenta los resultados del análisis estadístico para éste segmento, en él, se podría representar la presencia de los factores de riesgo mediante la presencia percibida en los siguientes ítems cuya respuesta en el cuestionario ha obtenido un valor igual o superior a 3,5. 
Tabla $N^{\circ} 2$ Análisis estadístico segmento trabajador electricista

\begin{tabular}{lc}
\hline \multicolumn{1}{c}{$\quad$ Ítem } & Valor Percibido \\
\hline RIESGO MECÁNICO & \\
Existencia de certificados para trabajo en altura (Caída de Altura) & \\
Condiciones de seguridad (Caída de Altura) & 3,95 \\
Inspecciones y controles de riesgo de carga (Riesgo de Transporte) & 3,71 \\
Ubicación optima(Aplastamientos por Máquinas y Equipos) & 3,64 \\
Existencia de métodos de manipulación(Aplastamientos por Máquinas y Equipos) & 3,63 \\
Estado de las máquinas de transmisión (Trasmisión de Fuerza) & 3,58 \\
Estado de los interruptores de emergencia (Trasmisión de Fuerza) & 3,55 \\
Eficiencia de los programas para trabajos en altura (Caída de Altura) & 3,55 \\
\hline RIESGO DISERGONÓMICO & 3,54 \\
Señalización de espacios (Espacio de Trabajo) & \\
\hline RIESGO ELÉCTRICO & 3,50 \\
Existencia de conexiones a tierra (Energía Estática) & \\
Existencia de normas de seguridad (Descargas Atmosféricas) & 3,77 \\
Estado de las instalaciones eléctricas (Descargas Atmosféricas) & 3,54 \\
\hline
\end{tabular}

Fuente: Elaboración propia.

En el Tabla $\mathrm{N}^{\circ} 3$ se presentan dichos ítems para cada segmento considerado, es decir aquellos que no sobrepasan la puntuación de 2,5 .

Tabla №3 Análisis estadístico para segmento trabajador electricista

\begin{tabular}{|c|c|}
\hline Ítem & Valor Percibido \\
\hline \multicolumn{2}{|l|}{ RIESGO FÍSICO } \\
\hline Existencia de controles para gestionar radiaciones (Radiaciones lonizantes) & 2,49 \\
\hline Elaboración de indicadores de ruido (Ruido Ocupacional) & 2,39 \\
\hline Efectos de las radiaciones no ionizantes (Radiaciones lonizantes) & 2,18 \\
\hline Realiza estudios de ruido ocupacional (Ruido Ocupacional) & 2,18 \\
\hline Ausentismo por efectos térmicos (Sensación Térmica) & 2,05 \\
\hline Efectos de las vibraciones sobre el trabajador (Vibraciones) & 2,02 \\
\hline Ausentismo por problemas auditivos (Ruido Ocupacional) & 1,98 \\
\hline Accidentes debidos a mala iluminación (lluminación Ocupacional) & 1,92 \\
\hline Accidentes debidos a las radiaciones (Radiaciones lonizantes) & 1,74 \\
\hline \multicolumn{2}{|l|}{ RIESGO QUÍMICO } \\
\hline Medidas de prevención de incendios (Inflamabilidad) & 2,46 \\
\hline Efectos de las sustancias corrosivas a trabajadores (Corrosión) & 2,45 \\
\hline Efectos de los químicos sobre los trabajadores (Toxicidad) & 2,04 \\
\hline Existencia de accidentes por incendios (Inflamabilidad) & 1,85 \\
\hline
\end{tabular}

Fuente: Elaboración propia, 2015 


\subsubsection{ANÁLISIS ESTADÍSTICO SEGMENTO SUPERVISOR HSEQ}

Como en el anterior análisis los ítems que presenten un valor igual a mayor a 3,5 hace perceptible la presencia del factor de riesgo, En la tabla $\mathrm{N}^{\circ} 4$ se presenta el análisis estadístico para el segmento.

Tabla $\mathbf{N}^{\circ} 4$ Análisis estadístico para segmentación supervisor HSEQ

\begin{tabular}{lc}
\hline \multicolumn{1}{c}{ Ítem } & $\begin{array}{c}\text { Valor } \\
\text { Percibido }\end{array}$ \\
\hline RIESGO BIOLÓGICO & 3,53 \\
Clasificación de desechos (Condiciones Bíoambiental) & 3,78 \\
\hline RIESGO MECÁNICO & 3,56 \\
Existencia de certificados para trabajo en altura (Caída de Altura) & 3,50 \\
Inspecciones y controles de riesgo de carga (Riesgo de Transporte) & 3,86 \\
Medidas para identificar equipos y herramientas inadecuadas(Caída de Objetos) & 3,69 \\
\hline RIESGO ELÉCTRICO & 3,61 \\
Existencia de conexiones a tierra (Energía Estática) & $-3,56$ \\
Existencia de normas de seguridad (Descargas Atmosféricas) & $-3,50$ \\
Existencia de procedimientos de trabajo (Contacto Eléctrico Directo) & - \\
Existencias de protocolos de seguridad(Quemaduras) & \\
Estado de las instalaciones eléctricas (Descargas Atmosféricas) & \\
\hline
\end{tabular}

Fuente: Elaboración propia.

En el Tabla $\mathrm{N}^{\circ} 5$, se presentan dichos ítems para cada segmento considerado, es decir aquellos que no sobrepasan la puntuación de 2,5.

Tabla $N^{\circ} 5$ Análisis estadístico para segmento supervisor HSEQ

\begin{tabular}{|c|c|}
\hline Ítem & Valor Percibido \\
\hline \multicolumn{2}{|l|}{ RIESGO FÍSICO } \\
\hline Existencia de controles para gestionar radiaciones (Radiaciones lonizantes) & 2,39 \\
\hline Elaboración de indicadores de ruido (Ruido Ocupacional) & 2,33 \\
\hline Efectos de las radiaciones no ionizantes (Radiaciones lonizantes) & 2,17 \\
\hline Ausentismo por problemas auditivos (Ruido Ocupacional) & 2,06 \\
\hline Realiza estudios de ruido ocupacional (Ruido Ocupacional) & 2,06 \\
\hline RIESGO BIOLÓGICO & 2,06 \\
\hline Efectos a los trabajadores (Agentes Patógenos) & 2,03 \\
\hline Ausentismo por efectos térmicos (Sensación Térmica) & 1,67 \\
\hline Accidentes debidos a mala iluminación (lluminación Ocupacional) & 1,53 \\
\hline \multicolumn{2}{|l|}{ RIESGO QUÍMICO } \\
\hline Efectos de las sustancias corrosivas a trabajadores (Corrosión) & 1,89 \\
\hline Mala manipulación de sustancia química (Oxido Reducción) & 1,83 \\
\hline Efectos de los químicos sobre los trabajadores (Toxicidad) & 1,53 \\
\hline Existencia de accidentes por incendios (Inflamabilidad) & 1,42 \\
\hline \multicolumn{2}{|l|}{ RIESGO BIOLOGICO } \\
\hline Efectos a los trabajadores (Agentes Patógenos) & 2,17 \\
\hline Contaminación en el lugar de trabajo (Condiciones Bíoambiental) & 1,75 \\
\hline \multicolumn{2}{|l|}{ RIESGO MECÁNICO } \\
\hline Ausentismo debido a caídas (Caída de Nivel) & 2,25 \\
\hline \multicolumn{2}{|l|}{ RIESGO DISERGONÓMICO } \\
\hline Equipos y herramientas óptimos (Movimientos Repetitivos) & 2,50 \\
\hline \multicolumn{2}{|l|}{ RIESGO PSICOSOCIAL } \\
\hline Ausentismo por insatisfacción (Síndrome del Quemado) & 2,47 \\
\hline Ausentismo laboral por estado de ánimo (Relaciones Interpersonales) & 2,25 \\
\hline
\end{tabular}

Fuente: Elaboración propia. 


\subsubsection{ANÁLISIS ESTADÍSTICO SUPERVISOR HSEQ}

Igual que en los casos anteriores, se presenta los estadísticos descriptivos del segmento supervisor HSEQ para el cuestionario Il de Evaluación del Sistema de Gestión de Seguridad y Salud Ocupacional debido a la existencia o mejora en los ítems cuya respuesta en el cuestionario ha sido igual o superior a 3,5. Los resultados se presentan en las siguientes tablas 22 y 23.

Tabla Nº6 Análisis estadístico para segmento supervisor HSEQ, sistema de gestión de seguridad y salud ocupacional

\begin{tabular}{lc}
\hline \multicolumn{1}{c}{ Ítem } & Valor Percibido \\
\hline Conocimientos (Programas) & 3,92 \\
Disminución de accidentes (Planes) & 3,86 \\
Exámenes médicos (Reglamentos / Normativas) & 3,81 \\
Difusión (Política de SSO) & 3,81 \\
Conocimiento (Manual de Funciones y Responsabilidades) & 3,78 \\
Eficacia (Programas) & 3,72 \\
Cumplimiento (Reglamentos / Normativas) & 3,69 \\
Actividades para garantizar la seguridad y salud (Misión) & 3,69 \\
Expectativa de crecimiento (Visión) & 3,69 \\
Existencia de Herramientas para cumplir (Planes) & 3,67 \\
Correspondencia (Política de SSO) & 3,67 \\
Aplicación de protocolos (Procedimientos) & 3,61 \\
Eficacia y eficiencia plan emergencia (Planes) & 3,61 \\
Claridad y definición de tareas (Procedimientos) & 3,58 \\
Conocimiento (Procedimientos) & 3,53 \\
Cooperación (Manual de Funciones y Responsabilidades) & 3,50 \\
\hline
\end{tabular}

Fuente: Elaboración propia.

Tabla N7 Análisis estadístico para segmento supervisor HSEQ, accidentabilidad y condiciones de salud de los trabajadores e impacto social

Ítem Valor Percibido

\begin{tabular}{lc}
\hline Existencia de medidas de mejora (Severidad) & 4,03 \\
Eficacia y eficiencia de acciones (Medidas Preventivas) & 4,00 \\
Indicadores de monitoreos (Ausentismo Accidentes y Enfermedades) & 3,97 \\
Efectividad de las investigación de accidente (Indemnizaciones) & 3,97 \\
Adecuación de turnos de trabajo (Horas Trabajadas) & 3,97 \\
Existencia de medidas preventivas y correctivas (Discapacidad) & 3,94 \\
Eficacia y eficiencia de planes (Medidas Preventivas) & 3,78 \\
Correspondencia precio valor de planes de emergencia (Medidas Preventivas) & 3,78 \\
Efectividad de las medidas de seguridad (Discapacidad) & 3,75 \\
\hline Existencia de indicadores (Frecuencia de Accidente) & 3,72 \\
Efectividad de los planes de contingencia (Daños Materiales) & 3,69 \\
Medidas preventivas (Horas Perdidas) & 3,67 \\
Calidad de servicio ofrecido por los aseguradores de riesgo (Medidas Preventivas) & 3,50 \\
\hline
\end{tabular}

Fuente: Elaboración propia. 
También en la tabla $\mathrm{N}^{\circ} 8$, se muestran los valores menos relevantes en los resultados de los ítems, es decir aquellos valores que obtuvieron valores igual 0 inferior a 2,5 para la variable Accidentabilidad y Condiciones de Salud de los Trabajadores e Impacto social. En el caso de la variable Sistema de Gestión de Seguridad y Salud Ocupacional, no se obtuvieron valores por debajo de 2,5 en ninguno de sus ítems.

Tabla N ${ }^{\circ} 8$ Análisis estadístico para segmento supervisor HSEQ, accidentabilidad y condiciones de salud de los trabajadores e impacto social

\begin{tabular}{l} 
Horas operativas perdidas (Severidad) \\
Frecuencia de discapacidad (Discapacidad) \\
Frecuencia de daños (Daños Materiales) \\
\hline Fuente: Elaboración propia, 2015 \\
3.4 DICUSION:
\end{tabular}

A continuación se relaciona la discusión en la identificación de los factores de cada riesgo percibidos por los encuestados (Trabajador Electricista y Supervisor HSEQ), estos son:

Factor Riesgo Físico: Para los dos grupos de encuestados, se puede interpretar que este factor no es relevante y podría interpretarse que es inexistente, Caso contrario se podría inferir lo siguiente:

$\checkmark$ Que la empresa no dispone de buenos controles para gestionar las radiaciones de tipo no ionizantes dentro de la organización.

$\checkmark$ Dado la inexistencia de cualquier tipo de radiación no ionizante los trabajadores no son afectados en absoluto por éstos.

$\checkmark \quad$ No se han registrado accidentes ni bajas laborales debido a las radiaciones no ionizantes.

$\checkmark$ La empresa no elabora indicadores de ruido, por lo tanto no se dispone de mecanismo de monitoreo de este agente para logra gestión de riesgo.

$\checkmark \quad$ No realiza estudios de ruido ocupacional. $\mathrm{Ni}$ tampoco se registra ausentismo y accidentes laborales relacionados con este. $\checkmark$ No se registran ausentismos ni accidentes laborales debido a temperaturas extremas de ningún tipo.

$\checkmark$ No hay evidencia de presencia del factor vibraciones ni se presentan evidencia de registros de trabajadores afectados por este agente.

$\checkmark \quad$ No se tiene registro de ambientes faltos de buena iluminación, debido a esto no se presentan registros de accidentes laborales por este agente.

Factor Riesgo Químico: Al igual que el anterior factor, puede interpretar que éste npes relevante y podría interpretarse que es inexistente, caso contrario se podria inferir lo siguiente: $\quad 2,44$

$\checkmark \quad$ No se registran accidentes per,2Bcendios debido al uso y manipulación de su,zjancias químicas inftamabtes.

$\checkmark \quad$ No se registran accidentes de lesiones en los trabajadores debido a la manipulación de sustancias químicas corrosivas.

$\checkmark$ No se presentan intoxicaciones en los trabajadores debido a la manipulación y mal manejo de sustancias químicas toxicas.

$\checkmark$ Los trabajadores electricistas, opinan que las medidas para prevenir los incendios no son las más adecuadas.

$\checkmark$ Los supervisores HSEQ, coinciden en afirmar que las sustancias oxídantes son manipuladas de forma adecuada.

Factor Riesgo Biológico: En este caso solo los supervisores de HSEQ presentan información en este factor, por lo que puede interpretar que éste es poco relevante, por lo tanto podría indicar que no existe presencia de éste en el ambiente laboral. En el caso de los valores de los trabajadores electricistas, estos se encuentran ubicados entre un rango menor de $3,5 \mathrm{y}$ mayor de 2,5. Caso contrario se podría inferir lo siguiente:

$\checkmark$ No se registran efecto de enfermedades profesionales en los trabajadores debido al contacto con agentes de tipo patógenos.

$\checkmark \quad$ No se presentan contaminación en los ambientes laborales que haya ameritado hospitalización de algún trabajador.

$\checkmark$ Al parecer la empresa posee un sistema de clasificación, depósito y disposición adecuado de los desechos sólidos en los lugares de trabajo. 
EVALUACION DEL IMPACTO DE LOS FACTORES DE RIESGO QUE GENERAN ENFERMEDADES PROFESIONALES Y ACCIDENTES LABORALES EN LAS PYMES DEL SECTOR ELÉCTRICO DE LA CIUDAD DE CÚCUTA-COLOMBIA

Gustavo Enrique Bohórquez Mantilla - Javier Mauricio García Mogollón

Factor Riesgo Mecánico:

$\checkmark$ Ambos encuestados afirman, que la empresa posee un procedimiento certificado para realizar trabajo en altura, el cual evita el riesgo de caídas de altura.

$\checkmark$ Ambos encuestados afirman, que la empresa realiza inspección y control de riesgo de caída de objetos en los equipos de izar y de transporte.

$\checkmark$ Los trabajadores electricistas indican que revisan las condiciones de seguridad cuando se realizan trabajos en altura.

$\checkmark$ Se dispone de una óptima ubicación para las máquinas y equipos, además de disponer de métodos de manipulación de éstos para así evitar riesgo de aplastamiento.

$\checkmark$ Se cuenta con un buen estado de las máquinas de transmisión de fuerza.

$\checkmark$ Los programas para trabajo en altura son eficientes y todos los dispositivos de emergencia se encuentran en condiciones óptimas.

$\checkmark$ Por su parte el Supervisor HSEQ, indica que las medidas empleadas para la identificación de los equipos y herramienta es inadecuada.

$\checkmark$ Por último, el supervisor de HSEQ, asevera que si se han presentado ausentismos de los trabajadores debido al riesgo de caída del mismo nivel.

Factor Riesgo disergonómico:

$\checkmark$ Los trabajadores electricistas indican que los espacios de trabajo están señalados.

$\checkmark$ Para los supervisores HSEQ, los equipos y herramientas deben de ser cambiados para evitar lesiones músculo-esquelética debido a movimientos repetitivos.

Factor Riesgo Eléctrico:

$\checkmark$ Para ambos encuestados, todos los equipos disponen de buena conexión a tierra de manera de evitar descargas por energía estática.

$\checkmark$ Ambos indican, que la empresa dispone de un buen estado de las instalaciones eléctricas y también de normas de seguridad adecuadas que evitan la ocurrencia de riesgos de descargas atmosféricas.

$\checkmark$ Los supervisores HSEQ, indican que la empresa dispone de buenos protocolos de seguridad para contrarrestar los riesgos de quemaduras por arco eléctrico. $Y$ también, se dispone de procedimientos de trabajo seguro para evitar el contacto eléctrico directo.

Factor Riesgo Psicosocial: En cuanto al factor psicosocial, debido a alto grado de complejidad y riesgo que presenta la labor desarrollada por los trabajadores del sector estudiado, es probable que éste factor sea poco conocido por los encuestados y por lo tanto este desconocimiento se ha pasado por alto y no se ha tomado en cuenta. En todo caso se muestra solo los resultados de los supervisores HSEQ.

$\checkmark$ No se han presentado ausentismo por insatisfacción en la labor realizada (síndrome del quemado) ni por mal estado de ánimo o mal clima laboral (relaciones interpersonales).

Con relación al Sistema de Gestión de Seguridad y Salud Ocupacional y a los indicadores de Accidentabilidad y Condiciones de Salud de los Trabajadores e Impacto Social se tiene lo siguiente:

Sistema de Gestión de Seguridad y Salud Ocupacional: Como se puede observar en los resultados de los 19 ítems que miden éste aspecto 16 resultaron relevantes para los supervisores HSEQ, es decir obtuvieron valor mayor o igual a 3,5.

$\checkmark$ La mayoría de los encuestados afirman que conocen los programas de seguridad e higiene que emplea la empresa, y estos son eficaces.

$\checkmark \quad$ Afirman que los planes emprendidos reducen los accidentes laborales, y se disponen de herramientas que permite lograr los objetivos trazados.

$\checkmark$ Se cumple con la reglamentación de los exámenes médicos y estos aseguran la salud de los trabajadores.

$\checkmark \quad$ Los trabajadores dan fiel cumplimiento a las reglas y normativas que la empresa posee.

$\checkmark$ La empresa dispone de un adecuado instrumento de comunicación y difusión de la política de seguridad e higiene de la organización y esta se corresponde con la realidad de la empresa.

$\checkmark$ Todos los trabajadores de la empresa están al tanto de sus funciones y responsabilidades al estar en su sitio de trabajo.

$\checkmark$ En la empresa se realizan actividades dirigidas a garantizar la seguridad y salud de los trabajadores 
dándole cumplimiento a la misión establecida por la organización.

$\checkmark \quad$ La empresa hace los esfuerzos necesarios para cumplir las expectativas de los trabajadores y la organización, tal como está establecido en la visión de la organización.

$\checkmark$ La empresa aplica adecuadamente los procedimientos de protocolos de seguridad establecidos por la organización para ejecutar las actividades y evitar accidentes.

$\checkmark$ La organización tiene establecido los planes de emergencia y contingencia y estos son eficaces y eficientes.

$\checkmark$ Los empleados conocen sus tareas y éstas están bien definidas y son claras y están dispuestas adecuadamente en los manuales de la organización.

$\checkmark$ Se fomentan el trabajo en equipo y la cooperación entre los trabajadores y está definida claramente en los manuales en el apartado de funciones y responsabilidades.

Indicadores de Accidentabilidad y Condiciones de Salud de los Trabajadores e Impacto Social:

Para este aspecto de la investigación de los 23 ítems del cuestionario II, 13 aspectos resultaron relevantes, es decir se obtuvieron valores iguales o superiores a 3,5 y 3 valores con resultados iguales o menor a 2,5 estos son:

$\checkmark$ La mayoría de los encuestados coinciden a afirmar que las medidas preventivas y correctivas de mejora adoptadas disminuyen la severidad de los accidentes laborales.

$\checkmark$ Se percibe en los encuestados que los planes de emergencia y contingencia implementadas en la organización son eficaces y efectivos, además se observa que estos planes se corresponden con el precio invertido y que la calidad del servicio ofrecido garantiza y asegura un riesgo aceptable en la organización.

$\checkmark$ Con relación al ausentismo y sus consecuencias, la organización emplea indicadores que permiten un monitoreo óptimo.

$\checkmark$ Se observa un efectivo procedimiento de investigación de accidente, que permite establecer un monto de indemnización justa para el trabajador. $\checkmark$ Las horas programadas en los turnos de trabajo son ergonómicas y confortables que permiten garantizar un clima organizacional óptimo.

$\checkmark$ Los encuestados consideran que las medidas emprendidas son tan efectivas que tienen un efecto positivo sobre la salud de los trabajadores y reduce las lesiones discapacitante. Esto también, es congruente con los resultados obtenidos en los valores menores o iguales a 2,5.

$\checkmark$ Con respecto a la frecuencia de ocurrencia de los accidentes, los encuestados afirman basados en los indicadores de gestión de la función de seguridad e higiene laboral, que éstos son bajos.

$\checkmark$ También se reporta que la efectividad de los planes emprendidos por la gerencia de la organización generan una menor cantidad de daños materiales. Esta afirmación también se corresponde con los resultados obtenidos en los valores menores e igual a 2,5 .

$\checkmark$ Por último, las medidas efectivas emprendidas por la gerencia generan menos cantidad de horas perdidas. Lo que indica que la severidad de los accidentes son bajos.

\section{CONCLUSIONES Y RECOMENDACIONES:}

De la presente investigación se pretenden las siguientes conclusiones que están directamente relacionadas con los objetivos específicos:

Se pudo observar que las PYMES del sector eléctrico de la ciudad de Cúcuta identifican los factores de riesgo que se presentan en el ambiente laboral, más sin embargo es necesario realizar actividades de sensibilización y de capacitación dirigida a los trabajadores del sector eléctrico con el fin de crear cultura organizacional en el autocuidado, debido a que

por exceso de confianza en las actividades realizadas diariamente se tienda a salta protocolos de seguridad, que pueden llegar a propiciar accidentes y enfermedades profesionales en los mismos. 
En cuanto a la estructura de gestión de seguridad y salud ocupacional, se evidencio que las PYMES del sector eléctrico de la ciudad de Cúcuta cuentan con una estructura de gestión de seguridad y salud ocupacional, donde los trabajadores conocen: los programas de seguridad e higiene que emplea la empresa, conocen las reglas y la normatividad, conocen los protocolos de seguridad establecidos por la organización, los planes de emergencia y contingencia.

Si bien es cierto que los trabajadores de las PYMES del sector eléctrico conocen la estructura de la seguridad y la salud ocupacional, es necesario que se implementen periódicamente acciones preventivas por medio de la capacitación de los gerentes y trabajadores que permitan mantener la gestión continua y la concientización a la prevención de accidentes y enfermedades profesionales.

En cuanto al impacto económico de los factores de riesgo, a la fecha no se han presentado accidentes, ni enfermedades profesionales en las PYMES del sector eléctrico estudiadas, más sin embargo la organización debe realizar la proyección anual de los costos que acarrearían la materialización de los riesgos que comúnmente se encuentran expuestos los trabajadores.

\subsection{Recomendaciones:}

Se proponen los lineamientos estratégicos de mejora organizacionales para minimizar el impacto de los factores de riesgos que generan accidentes y enfermedades profesionales las PYMES del sector eléctrico de la ciudad de Cúcuta, por medio de cuatro propuestas: concientización, sensibilización y motivación de los trabajadores de las PYMES del sector eléctrico, talleres de capacitación para la implementación de la gestión de seguridad y salud ocupacional para todos los niveles de la organización, implementación del plan estratégico y evaluación de la gestión.

Se hace necesario que las PYMES del sector eléctrico deban contratar conforme exige la ley la cantidad de vigías de salud ocupacional de acuerdo a la cantidad de trabajadores y seccionales de la empresa, debido a que en las visitas realizadas, algunas empresas por la cantidad de trabajadores que se manejaban, solo existía una persona encargada de velar por la seguridad de los trabajadores. Dicha situación impedía que la persona estuviera presente en todos los sitios de trabajo verificando la seguridad respectivamente.

\section{REFERENCIAS:}

Asociación Española de Normalización y Certificación, (2001). Norma Europea EN ISO 5349-1 medición y evaluación de la exposición humana a las vibraciones transmitidas por la mano. AENOR. España.

Chiavenato Idalberto (2006). Introducción a la teoría general de la administración $7^{\circ}$ Edición. México: McGraw - Hill.

Consejo Colombiano de Seguridad (CCS), (2014). Guía del sistema se seguridad, salud en el trabajo y ambiente para contratista. Colombia, Bogotá.

Federación de Aseguradores Colombianos (Fasecolda), (2013). Tasa de accidentalidad.

http://www.fasecolda.com/index.php/ramos Iriesgos-laborales/estadisticas-del-ramol. (Consulta: Enero 2015) 
Fondo de Riesgos Laborales (2012). Informe estadístico de accidentes laborales. http://fondoriesgoslaborales.gov.co/seccion linformacion-estadistica/2012.html, (Consulta: Junio 2014)

Freud R, David, (2003). Conceptos de administración estratégica novena edición. Pearson educación. Méxio.

García-M., A. M., Malagón-Sáenz, E., \& GarcíaMogollón, J. M. (2018). Modelos de negocios en pymes agroindustriales: desafíos en el siglo XXI. In Vestigium Ire, 11(2), 41-67. Recuperado a partir de http://revistas.ustatunja.edu.co/index.php/iv estigium/article/view/1549

Garcia Ponson, Norka R. (2010). Factores de riesgo ocupacional y trastornos de salud de los linieros de la empresa eléctrica de Yaritagua. Trabajo de grado de Especialista en Salud e Higiene Ocupacional. Universidad Centrooccidental "Lisandro Alvarado". Estado Yaracuy, Barquisimeto. Venezuela.

Gratton, Lynda. Estrategias de capital humano. Prentice Hall, Pearson educación, Madrid, 2001, página 161. España.

Harold Koontz, Heinz Weihrich, Enrrique mercado (1998). Administración, $8^{\circ}$ Edición. México. McGraw - Hill.

Hernandez, Fernandez y Baptista (1998). Metodología de la investigación. McGraw Hill. México

Instituto Colombiano de Normas y Técnicas y Certificación (ICONTEC), (2007). Norma técnica colombiana en seguridad y salud ocupacional (NTC - OHSAS 18001:2007). Colombia: Bogotá.
Instituto Colombiano de Normas y Técnicas y Certificación (ICONTEC), (2012). Guía para la identificación de los peligros y la valoración de los riesgos en seguridad y salud ocupacional (GTC 45). Colombia: Bogotá.

Instituto Colombiano de Normas y Técnicas y Certificación, Icontec (1994). Principios de ergonomía visual, iluminación para ambientes de trabajo en espacios confinados. Colombia: Bogotá.

Instituto Colombiano de Normas y Técnicas y Certificación, Icontec (2008). Sistemas de gestión de la calidad NTC ISO 9001:2008. Colombia: Bogotá.

Instituto Colombiano de Normas y Técnicas y Certificación, Icontec (2007). Requisitos de gestión en seguridad y salud ocupacional NTC OHSAS 18001:2007. Colombia: Bogotá.

Instituto Nacional de Seguridad e Higiene en el trabajo, (1982). Guía de buenas prácticas NTP 5: Identificación de productos químicos por etiqueta. Centro de investigación y asistencia técnica, Barcelona. España.

Instituto Nacional de Seguridad e Higiene en el trabajo, (2001). Guía de buenas prácticas NTP 614: Radiaciones ionizantes normas de protección. Centro de investigación y asistencia técnica, Barcelona. España.

Jaques, elliot. La organización requerida.

Ediciones granica, buenos aires, 2004

Ministerio de la protección social (2007). Manual comité paritario de salud ocupacional. Colombia: Bogotá.

Ministerio de la protección social, Comité Paritario de Salud Ocupacional, 2007, Bogotá. 
Ministerio de Minas y Energía (2008). Reglamento técnico de instalaciones eléctricas (RETIE). Resolución número 907018. Bogotá, Colombia.

Ministerio de trabajo de España (2000). Manual de riesgos psicosociales en el trabajo: teoría y práctica. España

Ministerio de trabajo y seguridad social, (1979). Resolución 2400 (22, mayo, 1979). Por la cual se establecen algunas disposiciones sobre vivienda, higiene y seguridad en los establecimientos de trabajo. Colombia: Bogotá.

Ministerio de trabajo y seguridad social, (1990). Resolución 1792 (3, mayo, 1990). Por la cual se establecen los valores permisibles para la exposición ocupacional al ruido. Colombia: Bogotá.

Ministerio de trabajo y seguridad social, (2012). Resolución 1409 (9, Agosto, 2012). Por la cual se establece el reglamento de seguridad para protección contra caídas en trabajo en altura. Colombia: Bogotá.

Mondelo, Pedro. Gregori Torada, Enrique. Uriz Comas, Santiago. Vilella Castejón, Emilio. Lacambra Bartolomé, Esther. Ergonomía 2. Confort y Estrés Térmico. Alfaomega -UPC. México. 2001. Pág. 109.

Mondelo, Pedro. Gregori Torada, Enrique. Uriz Comas, Santiago. Vilella Castejón, Emilio. Lacambra Bartolomé, Esther. Ergonomía 2. Confort y Estrés Térmico. Alfaomega -UPC. México. 2001. Pág. 62.
Moreira, M. (2006). La organización de la información para la gestión del conocimiento en las empresas. Recuperado de

http://www.gestiopolis1.com/recursos7/Doc s/ger/organizacion-de-la-informacion-parala-gestion-del-conocimiento.htm

Organización Iberoamericana de Seguridad Social (OISS), (2013). II encuesta nacional de condiciones de seguridad y salud en el trabajo en el sistema general riesgos laborales. Grafiq Editores SAS. Bogotá, Colombia.

Organización Internacional del Trabajo (OIT), (2013). Boletín sobre prevención de enfermedades profesionales. http://www.llo.org/safework/events/meeting s/WCMS_204931/lang--es/index.htm (Consulta, Noviembre 2014)

Organización mundial de la salud y la organización panamericana de la salud en las américas, (2007 a 2009). Caso de enfermedad y accidentalidad en las américas. http://www.paho.org/arg/index.php?option= com_content\&view=article\&id $=1155 \% 3$ Aop s-oms-estima-que-hay-770-nuevos-casosdiarios-personas-enfermedadesprofesionalesamericas\&catid $=332 \% 3$ Aarg02-prevenciny-control-de-enfermedades\&ltemid $=510$, (consulta, Noviembre 2014)

Organización panamericana de la salud (2002). La salud en las américas. Washington. 
Piedrahíta, Hugo (2002). La muerte en el trabajo. Revista facultad nacional de la salud pública volumen 20. Universidad de Antioquia. Colombia.

Ramirez Lorenzo, Luz Adriana (2007). Características epidemiológicas de las muertes accidentales en escenarios laborales en Bogotá. Colombia.

Robbins, S. y Coulter, (2005). Administración octava edición. Person educación. México.

Rodriguez y Rodriguez, Sergio Hernandez y Rodriguez, (2006). Introducción a la Administración $4^{\circ}$ Edición. México. McGraw - Hill.

Sampieri Hernández Roberto y Collado Fernández Carlos (2006). Metodología de la investigación (4 ed.). México. McGraw Hill.

Selltiz, Jahoda, Deutsch y Cook (1980) Métodos de investigación en las relaciones sociales. Madrid: Rialp. Existe una versión similar de este texto, cuyos autores son Selltiz, Wrightsman, Deustch y Cook.

Serrano Duran, José Manuel (2011). Análisis y gestión del riesgo en el mantenimiento de un sistema eléctrico, caso de una subestación de alta tensión. Trabajo de grado en maestro en ciencias en ingeniería de sistemas. México.

Tamayo y Tamayo (1987). El proceso de investigación científica. México: Limusa.

Tamayo y Tamayo, Mario, (1998). Diccionario de la investigación científica. Limusa, México.
Tellez García, Ingrid Carolina, (2009). Causas de los accidentes mortales en trabajo en altura. Trabajo de grado de especialista en seguridad ocupacional. Pontificia Universidad Javeriana. Colombia, Bototá.

Thompson/ strickland (2004), Administración estratégica. Textos y casos ( $13^{\circ}$ edición). México. Mc Hill Latinoamericana.

Vargas Porras, Paola Andrea, (2012). Caracterización demográfica y ocupacional de los casos de lesiones osteomusculares de miembros superiores y región lumbar. Trabajo de grado en Magister en salud y seguridad en el trabajo. Universidad nacional. Colombia, Bogotá.

Villacrés, Guillermo (2009). Gerencia estratégica. Trabajo de estudio. Caracas

Weiers, M. Weiers (1986). Investigación de mercados. México: Prentice Hill Hispanoamericana, S.A. 